\title{
Rapidly Progressive, Isolated Subretinal Leukemic Relapse: A Case Report
}

\author{
Thomas B. Gillette ${ }^{a}$ Michelle T. Cabrera ${ }^{b}$ Katherine Tarlock ${ }^{c}$ Claire E. Murphy ${ }^{d}$ \\ Karen M. Chisholm ${ }^{d, e}$ Andrew W. Stacey ${ }^{b}$ \\ ${ }^{a}$ Clinical and Translational Research, Seattle Children's Hospital, ${ }^{b}$ Department of Ophthalmology, University of \\ Washington, 'Department of Hematology-Oncology, Seattle Children's Hospital, dDepartment of Laboratory \\ Medicine, University of Washington, and ' Department of Laboratories, Seattle Children's Hospital, Seattle, WA, USA
}

\section{Established Facts}

- Relapse of acute leukemia may present as a subretinal infiltrate.

- Intraocular relapse may be isolated or occur in conjunction with bone marrow and/or central nervous system (CNS) relapse.

\section{Novel Insights}

- A serous retinal detachment in a patient with acute leukemia can rapidly progress to complete subretinal infiltration. Urgent biopsy should be considered for unexplained retinal detachments in these patients.

- Intraocular relapse may occur in a patient with no prior evidence of CNS disease and no evidence of CNS disease at presentation.

\section{Keywords}

Subretinal infiltrate $\cdot$ Leukemia $\cdot$ Ocular relapse . Enucleation · Optic nerve infiltration - Serous retinal detachment - Fine-needle aspiration choroidal biopsy . Fine-needle aspiration

\footnotetext{
Abstract

Purpose: The aim of this paper is to describe a case of relapsed pediatric acute lymphoblastic leukemia (ALL) presenting as a rapidly progressive subretinal infiltrate, as diagnosed by ultrasound-guided fine needle aspiration (FNA). Methods: We conducted a clinical pathological retrospective chart review. Results: Eleven months after documented
}

remission of T-cell ALL while on maintenance therapy, this 17-year-old patient presented with acute open angle glaucoma in the right eye. B-scan ultrasonography suggested total retinal detachment. Eight weeks later, based on routine cerebrospinal fluid analysis, the patient was diagnosed with central nervous system relapse of T-cell ALL. Repeat B-scan 1 week later showed a new hyperechoic subretinal mass. FNA of the mass confirmed leukemic infiltrate. The involved eye was enucleated, demonstrating leukemic cells throughout the subretinal space, choroid, and the optic nerve. Following hematopoietic stem cell transplant, the patient continues to maintain bone marrow remission 5 months after enucleation without involvement in the opposite eye. Conclusion: Retinal detachment in any patient with a history of

\section{KARGER}

(C) 2017 S. Karger AG, Basel

E-Mail karger@karger.com

www.karger.com/oop
Thomas Bradford Gillette, MD

908 Jefferson St., 7th Floor

Seattle, WA 98104 (USA)

E-Mail tbg8@uw.edu 
leukemia should raise the possibility of relapse and may warrant aspiration/biopsy if other means of diagnosing relapse are inconclusive. Subretinal infiltrate may progress rapidly and prompt diagnosis is paramount to tailoring therapy and preserving vision.

(c) 2017 S. Karger AG, Basel

\section{Introduction}

Acute lymphoblastic leukemia (ALL) is the most prevalent cancer in children. Advances in diagnosis and treatment have increased the survival rate from less than $10 \%$ in the 1960 s to approximately $85-90 \%$ with contemporary treatments [1]. Ocular manifestations of pediatric ALL may be the presenting sign of the disease or may signify leukemic recurrence. The prevalence of ocular findings in pediatric ALL patients has been reported at 15$20 \%[2,3]$. The rate of ocular involvement at relapse has been reported at $2.2 \%$ and may represent a first relapse, either in isolation or combination with relapse at another site, or a second relapse [4].

Ophthalmic manifestations of leukemia can be divided into two distinct categories: primary infiltration by neoplastic cells, and secondary involvement from hematologic abnormalities. Primary infiltration occurs in the orbit (presenting as proptosis, lid edema, or chemosis) [5], the anterior segment (presenting as corneal edema, hypopyon, and posterior synechia) [6], the posterior segment (retinal and choroidal infiltrates), and the optic nerve [7]. The choroid is the most commonly infiltrated anatomic location within the eye and may result in a markedly thickened choroid with concurrent serous retinal detachment [8]. Secondary involvement of the eye is most commonly visualized in the retina with angiopathy, venous dilation and tortuosity, hemorrhages, cotton wool spots, central retinal vein occlusion, neovascularization, and microaneurysms [9].

Intraocular relapse may be isolated or occur in conjunction with bone marrow and/or central nervous system (CNS) relapse. Ridgway et al. [10] found a high correlation between CNS leukemia and ocular invasion, where among acute leukemia patients with ocular invasion, 27 of 29 had concurrent CNS involvement. Similar results were seen in relation to bone marrow involvement with 30 of the 52 children referred for "ocular abnormalities" demonstrating concurrent bone marrow relapse. Of those who did not have bone marrow involvement at the time, most relapsed several months after the recognition of ocular involvement [10]. Ocular relapse of ALL can be a clinical diagnosis but may require diagnostic biopsy of the iris, vitreous fluid, or choroid for confirmation. With respect to treatment, Sommervaille et al. [4] found that intensive chemotherapy regimens were associated with favorable outcomes, whereas when treated with less intensive chemotherapy or when the relapse occurred on therapy, outcome was poor.

We report a case of relapsed childhood T-cell ALL (TALL) with subretinal leukemic infiltrate in the setting of normal cerebrospinal fluid (CSF). Relapse presented as a rapidly progressive retinal detachment during active treatment with intrathecal chemotherapy and was diagnosed by fine-needle aspiration (FNA) under ultrasound guidance.

\section{Case Report}

A 17-year-old male with a past medical history significant for developmental delay, seizure disorder, and hydrocephalus status post ventriculoperitoneal shunt, was diagnosed with T-ALL, negative for CNS involvement. He entered complete remission after induction chemotherapy with dexamethasone, vincristine, daunorubicin, pegasparaginase, and intrathecal methotrexate (MTX). He then proceeded to post-induction chemotherapy and continued to receive high-dose chemotherapy, including CNS-directed therapy such as high-dose MTX and frequent intrathecal chemotherapy. Approximately 11 months following remission, he presented during routine maintenance chemotherapy with a 3-day history of right eye redness and swelling.

His past ocular history was remarkable for known bilateral optic atrophy and a baseline visual acuity of 20/400 in both eyes secondary to hydrocephalus, although prior exams were limited due to the patient's developmental delay. On the day of presentation, he was 4 months into maintenance therapy, which included dexamethasone, vincristine, mercaptopurine, oral MTX, and intrathecal MTX. Lumbar puncture showed an elevated opening pressure of $54 \mathrm{~mm} \mathrm{Hg}$, prompting evaluation with a computed tomography (CT) shunt series. No abnormality was identified in the ventricular system or shunt tubing; however, the CT was remarkable for a new irregular hyperdensity in the posterior segment of the right globe (Fig. 1a). Monthly CSF cytology evaluations prior to this presentation, at presentation, and 3 weeks later were all unremarkable, with no leukemic blasts or suspicious appearing cells.

On presentation to the ophthalmology clinic, his monocular visual acuity was difficult to assess due to poor cooperation, although no light perception of the right eye was suspected. His intraocular pressures were $71 \mathrm{~mm} \mathrm{Hg}$ in the right eye and $22 \mathrm{~mm}$ $\mathrm{Hg}$ in the left. Slit-lamp exam of the right eye showed $3+$ conjunctival injection, microcystic corneal edema, and enlarged iris vessels, with no view to the posterior segment. The exam of the left eye was remarkable for a pale optic nerve on dilated fundus exam. B-scan ultrasonography of the right eye appeared to show a total retinal detachment with mobile serous fluid under the retina (Fig. 1b). The patient's elevated intraocular pressure was treated topically and he was referred to the retina and ocular oncology service. 

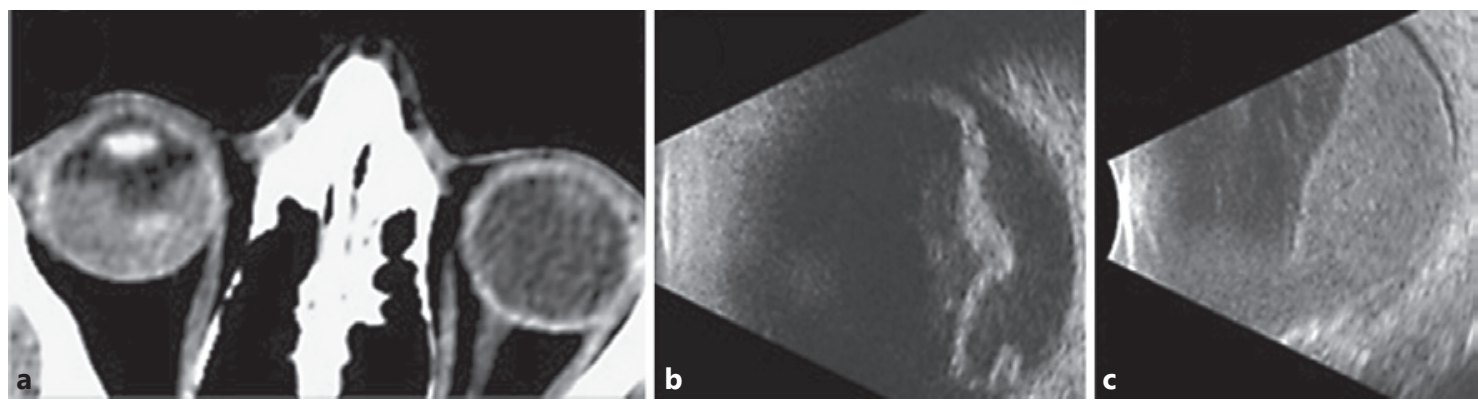

Fig. 1. a CT scan on initial presentation shows new irregular hyperdensity in the posterior chamber of the right globe. $\mathbf{b}$ B-scan ultrasonography of the right eye on initial presentation shows a total retinal detachment without a rhegmatogenous component and with mobile serous fluid under the retina. c B-scan ultrasonography of the right eye 9 weeks after initial presentation shows subretinal mass.

Fig. 2. a Intraoperative B-scan ultrasonography showing subretinal mass and fineneedle aspiration (FNA) needle (red arrow) in the subretinal space. b Blast cell with adjacent red blood cells on WrightGiemsa stain from FNA. The blasts had increased nuclear-to-cytoplasmic ratios, irregular nuclear contours, variably prominent nucleoli, basophilic cytoplasm, and perinuclear hofs.

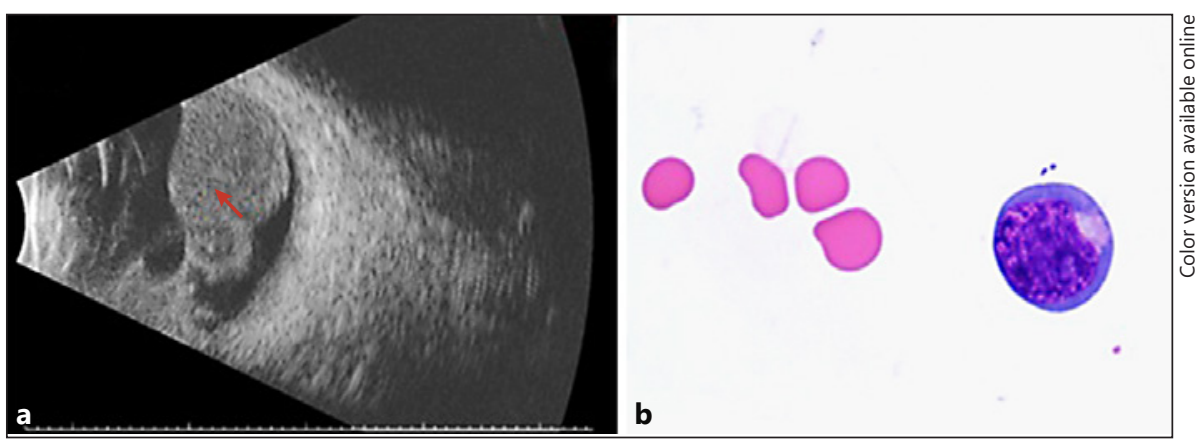

Unfortunately, at this time, the patient developed headaches, lethargy, and altered mental status. Imaging showed shunt malfunction with acute obstruction, and the patient was taken for neurosurgical shunt replacement. Further specialist consultation and ophthalmic testing were necessarily deferred during these treatments. Testing of the CSF and bone marrow continued to show no signs of leukemic relapse during treatment of the above. Eight weeks after initial presentation of eye complaints, and during course 3 of maintenance therapy with intrathecal MTX, a routine lumbar puncture showed 1,255 white blood cells in a setting of 75 red cells, and pathologic examination revealed numerous blasts. Bone marrow evaluation was negative for any evidence of measurable disease and a diagnosis of CNS relapse of T-ALL was made. One week later, the patient presented to the ocular oncology service and a repeat $\mathrm{B}$-scan ultrasound showed dramatic progression of the serous subretinal fluid, which had evolved into a hyperechoic subretinal mass (Fig. 1c). In addition, there was a large $(10 \times$ $5 \mathrm{~mm}$ ) patch of scleromalacia visible on the inferior aspect of the globe, which was not previously present. At this time, the patient had recovered from his previous systemic issues and, prior to initiation of re-induction therapy, underwent a FNA of the subretinal mass to direct medical and radiation treatments. This was done under ultrasound guidance as there was no view to the posterior segment (Fig. 2). The subretinal fluid analysis revealed an abnormal immature T-cell population comprising $22.5 \%$ of white cells, confirming subretinal leukemic infiltration.
Following completion of re-induction therapy, the patient maintained bone marrow remission and achieved CNS remission as well. Given the end-stage glaucoma, no light perception vision, intraocular ALL relapse, and concern for scleral melt and spread of the intraocular disease, an enucleation was performed once the patient had recovered from re-induction chemotherapy. The right eye enucleation (Fig. 3) demonstrated leukemic cells throughout the subretinal space, choroid, and the optic nerve.

The patient went on to receive a hematopoietic stem cell transplant, with conditioning that included total body and cranial radiation with a boost to the right orbit.

\section{Discussion}

Retinal detachment secondary to subretinal leukemic infiltration is a rare complication of acute leukemias. Amongst 11 children with acute leukemia and ocular involvement over a 6-year period, Reddy and Menon [5] documented only one with retinal detachment. Interestingly, this same patient was the only case to have a documented relapse during the study, which occurred in the CNS. In a study of 17 cases of intraocular relapse as a first 


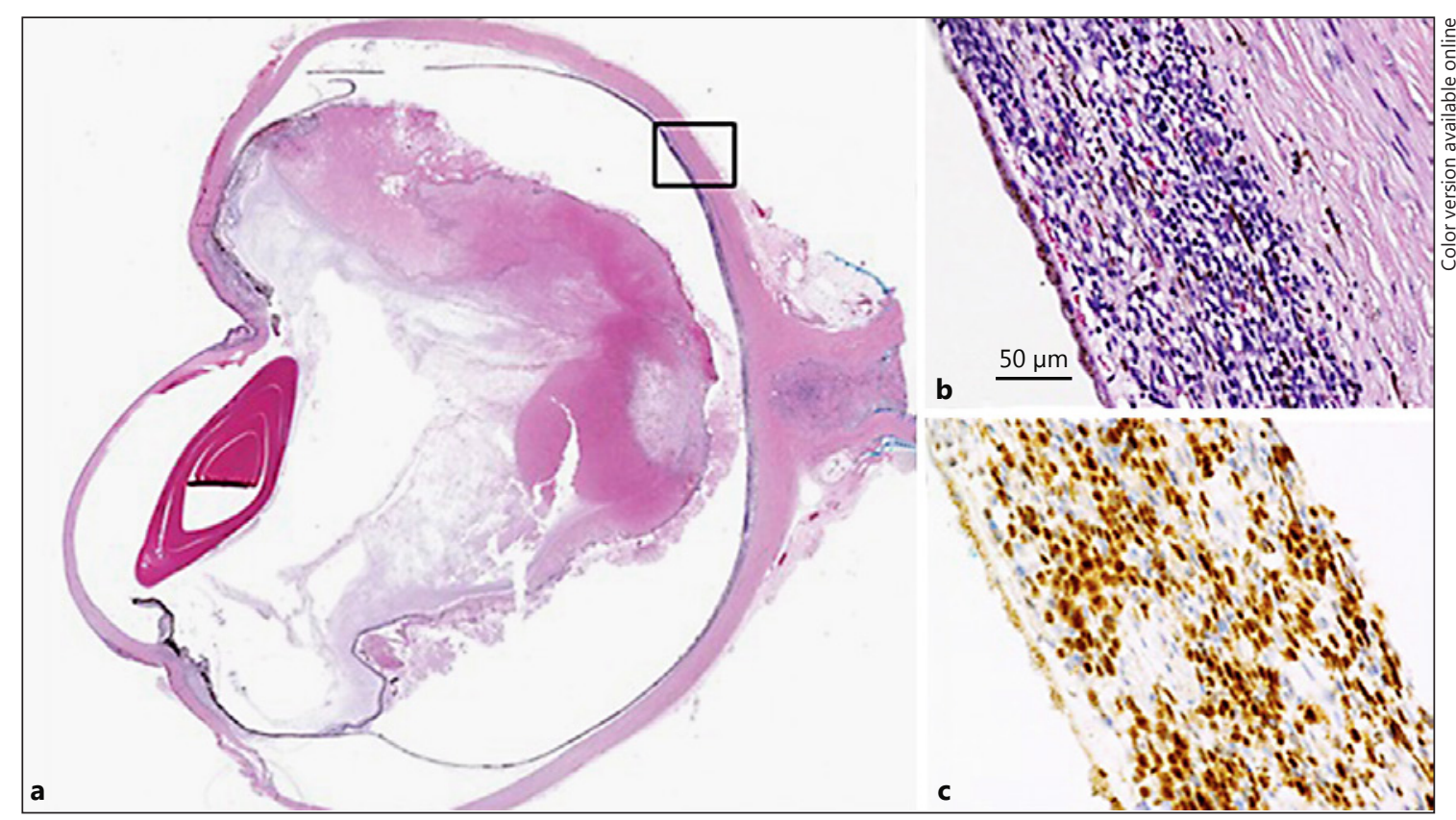

Fig. 3. Right eye enucleation. a Hematoxylin and eosin whole slide scan ( $0.5 \times$ magnification) of full cross section of eye including anterior chamber and lens on the left and optic nerve on the right. The vitreous chamber is involved by a necrotic and hemorrhagic neoplasm. Retinal detachment is present in most of the posterior aspect, with necrotic cells in the subretinal space. b Hematoxylin and eosin stain at $400 \times$ magnification from region highlighted by black box in a demonstrates choroid surfaced by retinal pigment epithelium. In this posterior region of the eye, there is retinal detachment. Within the choroid, there are increased large atypical lymphocytes with higher nuclear-to-cytoplasmic ratios and smoother chromatin. Pigmented melanocytes are also identified in the choroid and sclera. c TdT immunohistochemical stain at $400 \times$ magnification highlights the nuclei of the lymphoblasts within the choroid underlying the retinal pigmented epithelium. relapse of childhood ALL, Somervaille et al. [4] identified 4 cases that involved the retina and choroid. Two of the 4 occurred while on maintenance therapy and both subsequently passed away secondary to medullary relapse, 8 and 4 months after the first relapse. The median latency of ocular relapse following cessation of maintenance therapy among these 4 patients was 11.75 months, significantly longer than the 4-month latency period found for the entire first-relapse cohort; a duration similar to that seen at other extramedullary sites $[4,11]$.

We report a case of an unexplained retinal detachment in a patient with T-ALL. He was in remission on maintenance chemotherapy, including intrathecal MTX, with no prior history of CNS disease and no evidence of leukemic relapse in either the bone marrow or the CNS at the time of initial presentation. CNS relapse was subsequently diagnosed 8 weeks later. This case demonstrates the sobering truth that any unexplained retinal detachment in a patient with acute leukemia must raise warning flags, no matter if the leukemia is active or in remission, and regardless of treatment status. Such a finding should be considered a leukemic relapse until proven otherwise. If alternative methods to identify ALL relapse fail, such as blood work, lumbar puncture, and bone marrow biopsy, aspiration or biopsy of the subretinal fluid is warranted and may guide treatment decisions.

It has been suggested that the eye represents a "sanctuary" site in acute leukemia, harboring leukemic blast cells in the iris, choroid, or optic nerve that are suppressed but not eradicated by chemotherapy [12-14]. It has been postulated that harbored leukemic cells multiplying within the retina may impair normal retinal fluid resorption leading eventually to widespread serous retinal detachment [15]. Our case suggests that systemic chemotherapy and even intrathecal treatment may not be sufficient to prevent subretinal disease. The effects of subretinal relapse can be quickly progressive and visually catastrophic, as demonstrated in this case. Subretinal ALL relapse can quickly lead to prolonged retinal detachments, neovascular glaucoma, and a blind painful eye. It is important, therefore, to identify these relapses early and alter treatment to better target the subretinal space. In this case, the 
subretinal mass grew aggressively despite active chemotherapy and intrathecal treatment. Therefore, in cases of intraocular relapse of ALL, we recommend aggressive treatment with radiation if possible. As systemic treatments improve and more patients survive ALL, these types of ocular recurrences will likely increase and those who take care of patients with ALL should be aware of this presentation and the poor visual and systemic prognosis associated with it.

\section{Acknowledgements}

This research was funded in part by an unrestricted grant to the Department of Ophthalmology at the University of Washington from Research to Prevent Blindness.

\section{Statement of Ethics}

The institutional review board at Seattle Children's Hospital approved this study protocol as research not involving human subjects. Verbal informed consent was obtained from the patient's family.

\section{Disclosure Statement}

None of the authors have any financial conflicts of interest to disclose.

\section{References}

1 Hunger SP, Mullighan CG: Acute lymphoblastic leukemia in children. N Engl J Med 2015;373:1541-1552.

2 Russo V, Scott IU, Querques G, Stella A, Barone A, Delle Noci N: Orbital and ocular manifestations of acute childhood leukemia: clinical and statistical analysis of 180 patients. Eur J Ophthalmol 2008;18:619-623.

3 Reddy SC, Jackson N, Menon BS: Ocular involvement in leukemia - a study of 288 cases. Ophthalmologica 2003;217:441-445.

4 Somervaille TC, Hann IM, Harrison G, Eden TO, Gibson BE, Hill FG, Mitchell C, Kinsey SE, Vora AJ, Lilleyman JS; MRC Childhood Leukaemia Working Party: Intraocular relapse of childhood acute lymphoblastic leukaemia. Br J Haematol 2003;121:280-288.

5 Reddy SC, Menon BS: A prospective study of ocular manifestations in childhood acute leukaemia. Acta Ophthalmol Scand 1998;76: 700-703.
6 Yamamoto-Ogasawara A, Asakawa M, Yoshino K, Nagamoto T, Inoue M, Hirakata A: Anterior complications in case of recurrent acute lymphoblastic leukaemia diagnosed by biopsy of aqueous humour. Clin Exp Ophthalmol 2009;37:739-741.

7 Camera A, Piccirillo G, Cennamo G, Tranfa F, Rosa N, Frigeri F, Martinelli V, Rotoli B: Optic nerve involvement in acute lymphoblastic leukemia. Leuk Lymphoma 1993;11: 153-155.

8 Bajenova NV, Vanderbeek BL, Johnson MW: Change in choroidal thickness after chemotherapy in leukemic choroidopathy. Retina 2012;32:203-205.

9 Talcott KE, Garg RJ, Garg SJ: Ophthalmic manifestations of leukemia. Curr Opin Ophthalmol 2016;27:545-551.

10 Ridgway EW, Jaffe N, Walton DS: Leukemic ophthalmopathy in children. Cancer 1976;38: 1744-1749.
11 Lawson SE, Harrison G, Richards S, Oakhill A, Stevens R, Eden OB, Darbyshire PJ: The UK experience in treating relapsed childhood acute lymphoblastic leukaemia: a report on the medical research council UKALLR1 study. Br J Haematol 2000;108:531-543.

12 Ninane J, Taylor D, Day S: The eye as a sanctuary in acute lymphoblastic leukaemia. Lancet 1980;1:452-453.

13 Schmiegelow K, Scherfig E, Prause JU, Jensen OA: Isolated leukemic choroidal relapse in a child with acute lymphoblastic leukemia one year off therapy, diagnosed through transvitreal retino-choroidal biopsy. Acta Ophthalmol (Copenh) 1988;66:33-37.

14 Nagpal MP, Mehrotra NS, Mehta RC, Shukla CK: Leukemic Optic Nerve Infiltration in a Patient with Acute Lymphoblastic Leukemia. Retin Cases Brief Rep 2016;10:127-130.

15 Primack JD, Smith ME, Tychsen L: Retinal detachment in a child as the first sign of leukemic relapse: histopathology, MRI findings, treatment, and tumor-free follow up. J Pediatr Ophthalmol Strabismus 1995;32:253-256. 